\title{
The use of matrigel has no influence on tumor development or PET imaging in FaDu human head and neck cancer xenografts
}

Frederikke P. Fliedner ${ }^{1}$, Anders E. Hansen ${ }^{1,2}$, Jesper T. Jørgensen ${ }^{1}$ and Andreas Kjær ${ }^{1 *}$

\begin{abstract}
Background: In preclinical research Matrixgel ${ }^{\mathrm{TM}}$ Basement Membrane Matrix (MG) is used frequently for the establishment of syngeneic and xenograft cancer models. Limited information on its influence on parameters including; tumor growth, vascularization, hypoxia and imaging characteristics is currently available. This study evaluates the potential effect of matrigel use in a human head and neck cancer xenograft model (FaDu; hypopharyngeal carcinoma) in NMRI nude mice. The FaDu cell line was chosen based on its frequent use in studies of cancer imaging and tumor microenvironment.
\end{abstract}

Methods: NMRI nude mice $(n=34)$ were divided into two groups and subcutaneously injected with FaDu cells in medium either including $(+M G)$ or excluding matrigel $(-M G)$. In sub study I seven mice from each group ( $+M G, n=7 ;-M G, n=7)$ were ${ }^{18} \mathrm{~F}$ - fluorodeoxyglucose $\left({ }^{18} \mathrm{~F}-\mathrm{FDG}\right)$ PET/CT scanned on Day 5, 8, 12, 15, and 19. In sub study II ten mice from each group ( $+M G, n=10 ;-M G, n=10$ ) were included and tumors collected for immunohistochemistry $(\mathrm{IHC})$ analysis of tumor microenvironment including; proliferation ratio, micro vessel density, average vessel area, hypoxia, nuclear density, and necrosis. Tumors for $\mathrm{IHC}$ were collected according to size $\left(200-400 \mathrm{~mm}^{3}, 500-700 \mathrm{~mm}^{3}, 800-1100 \mathrm{~mm}^{3}\right)$.

Results: FDG uptake and tumor growth was statistically compatible for the tumors established with or without MG. The IHC analysis on all parameters only identified a significantly higher micro vessel density for tumor size 500-700 $\mathrm{mm}^{3}$ and 800-1100 $\mathrm{mm}^{3}$ and average vessel area for tumor size 500-700 $\mathrm{mm}^{3}$ in the -MG group. Comparable variations were observed for tumors of both the $+M G$ and $-M G$ groups. No difference in tumor take rate was observed between groups in study.

Conclusions: Matrigel did not affect tumor growth or tumor take for the FaDu xenograft model evaluated. Tumors in the -MG group displayed increased angiogenesis compared to the +MG tumors. No difference in ${ }^{18}$ F-FDG PET uptake for tumors of different groups was found. Based on these observations the influence of matrigel on tumor imaging and tumor microenvironment seems minor for this particular xenograft model.

Keywords: Matrigel, FaDu, Xenograft, PET imaging, Tumor development, Hypoxia, MVD, Cancer, FDG-PET, Molecular imaging

\footnotetext{
* Correspondence: akjaer@sund.ku.dk

${ }^{1}$ Department of Clinical Physiology, Nuclear Medicine \& PET and Cluster for

Molecular Imaging, Rigshospitalet and University of Copenhagen,

Blegdamsvej 9, 2100 Copenhagen, Denmark

Full list of author information is available at the end of the article
} 


\section{Background}

Human cancer xenografts in immunodeficient mice are widely used in cancer research and provide vital models for the study of tumor growth, tumor development, and the response to therapy in preclinical research. Several human cancer cell linescan be succesfully implanted onto immune deficient mouse models, but variations in take rate and growth of solid tumors makes their use challenging. Matrixgel ${ }^{\mathrm{TM}}$ Basement Membrane Matrix (Matrigel or MG) is commonly used to improve tumor take and growth [1]. Matrigel was originally extracted from connective tissue and research from the last century has shown that the extracts form matrix structures, and provide surrounding cells with substrates for growth promotion and development [1-3]. The reconstructed basement membrane complex includes; laminin, growth factors, entactin, and type IV collagen [3-5]. Studies have evaluated the advantages of MG for various cell lines [6-9] and generally it has been found to improve tumor take and growth. No change in tumor development and microenvironment is stated in these studies and reviews $[4,8,9]$. Isolated constituents from MG have been tested for impact on cell growth. However, no single substance was identified as the main mediator of effects $[1,10]$. MG hereby lacks a fully defined impact on tumor growth, which could be a possible source of error in translation. By legislation the number of animals in preclinical research must be kept as low as possible while maintaining adequate power of studies. Enhancement of tumor growth by including MG can be used to decrease the number of animals in a study, but could also be a potential source of error from a translational perspective. ${ }^{18} \mathrm{~F}-\mathrm{FDG} \mathrm{PET} / \mathrm{CT}$ is clinically used for head and neck cancer in relation to staging, therapy planning and response to therapy [11]. FaDu head and neck cancer xenograft models are widely used for studies of PET imaging, tumor microenvironment, and radiation therapy in preclinical research [12-14]. The description of MG use for tumor inoculation in studies is not always specified. Accordingly, an understanding of possible impact of MG use is of great importance. The aim of this study was to investigate the influence of MG use, on tumor and imaging characteristics of FaDu hypopharyngeal carcinoma cells inoculated subcutaneously on NMRI nude mice.

\section{Methods}

\section{Tumor model}

All experimental procedures were approved by the Danish Animal Welfare Council, the Danish Ministry of Justice. Dulbecco's Modified Eagle Medium were supplemented with $10 \%$ Fetal Calf Serum (FCS) and $1 \%$ penicillinstreptomycin for growth of FaDu cells in culture flaks until confluency, retained in $5 \% \mathrm{CO}_{2}$ incubator at $37^{\circ} \mathrm{C}$. Six weeks old female NMRI nude (Naval Medical Research
Institute) mice were purchased from Taconic Europe (Borup, Denmark). After one week of adaptation, 34 animals were inoculated with FaDu tumor cells subcutaneously on both left and right flank. Half of the mice $(n=17)$ were injected with a suspension for each tumor consisting of $2.5 \times 10^{6} \mathrm{FaDu}$ cells in $50 \mu \mathrm{L}$ Dulbecco's Modified Eagle Medium (Life Technologies, Carlsbad, CA, USA) (-MG group; $n=17$ ). For + MG group $(n=17) 50 \mu \mathrm{L}$ Matrixgel $^{\mathrm{Ts}}$ Basement Membrane Matrix (BD Biosciences, San Jose, CA, USA) was added and a total volume of $100 \mu \mathrm{L}$ containing $2.5 \times 10^{6} \mathrm{FaDu}$ cells injected. Tumor size and weight were measured continuously from day 5 post implant to follow the development of tumors and monitor the health of the mice. Mice were housed in IVC rack from Techniplast in Type III SPF cages with 8 mice in each cage. Purified water and chow food was available ad libitum for mice unless anything else is described.

\section{Group determination}

Of the 34 mice included in study; 14 mice (+MG, $n=7$; -MG, $n=7$ ) were randomized to sub study I where all mice were ${ }^{18} \mathrm{~F}$ - fluorodeoxyglucose $\left({ }^{18} \mathrm{~F}\right.$-FDG) PET/CT scanned on Day $5,8,12,15$, and 19 . In sub study II that included ten mice from the + MG group $(n=10)$ and ten mice from the $-\mathrm{MG}$ group $(n=10)$, tumors were collected when reaching predetermined sizes $\left(200-400 \mathrm{~mm}^{3}, 500-\right.$ $700 \mathrm{~mm}^{3}, 800-1100 \mathrm{~mm}^{3}$ ) for immunohistochemistry (IHC) analysis of tumor characteristics.

\section{Volume determination}

Tumor volume determination with external caliper was made by measuring the greatest longitudinal diameter and transverse diameter. Tumor volume was then calculated by following ellipsoid equation $[15,16]$ :

$$
\begin{aligned}
\text { Tumor Volume }=\pi & \cdot\left(\frac{\text { longitudinal diameter }+ \text { transverse diameter }}{2}\right)^{3} \\
& \cdot \frac{1}{6}
\end{aligned}
$$

Tumor volumes determined from ${ }^{18}$ F-FDG PET/CT were generated by manually drawing regions of interest (ROIs) to cover the entire tumor by numerous tomographic voxels, and summation of these defined the 3D tumor volume.

\section{${ }^{18}$ F-FDG PET/CT imaging - sub study I}

Mice were injected via the tail vein with a mean activity of $8.90 \pm 1.55 \mathrm{MBq}$ (Mean $\pm \mathrm{SD}$ ) ${ }^{18} \mathrm{~F}$-FDG in $0.2 \mathrm{~mL}$ $0.9 \%$ isotonic saline solution. Prior to injection all mice were fasted for approximately $12 \mathrm{~h}$ to minimize the variation in ${ }^{18}$ F-FDG uptake [17]. For injection, distribution, and scanning, all mice were kept anaesthetized with $3 \%$ sevoflurane (Abbott Scandinavia AB, Solna, Sweden) mixed 
with $35 \% \mathrm{O}_{2}$ and $65 \% \mathrm{~N}_{2}$. Body temperature was kept stable by external heating device when anaesthetized, and positioned on a heating pad during scan. ${ }^{18}$ F-FDG PET/CT imaging was performed on Siemens Inveon ${ }^{\circ}$ Small Animal Scanner (Siemens Medical Systems, PA, USA). The protocol included a five minute PET scan followed by a CT scan with attenuation correction to be used for reconstruction. Reconstruction of PET scans were performed using maximum a posteriori (MAP) reconstruction algorithm (voxel size: $0.815 \times 0.815 \times 0.796 \mathrm{~mm}$; resolution $(\mathrm{FWHM}$ ) $1.2 \mathrm{~mm}$ ). Reconstructed images were analyzed with Inveon Research Workspace software (Siemens Medical Systems, PA, USA). Tracer uptake was determined as mean and maximum $\%$ injected dose pr. gram of tumor (\%ID/g) $\left(1\right.$ gram per $\left.\mathrm{cm}^{3}\right)$, and mean and maximum standardized uptake value (SUV), corrected for decay.

\section{Tumor microenvironment - sub study II}

Tumors were collected when reaching a size of 200$400 \mathrm{~mm}^{3}$ (+MG; $n=6$, -MG; $\left.n=5\right), 500-700 \mathrm{~mm}^{3}$ (+MG; $n=7,-\mathrm{MG} ; n=6)$, and $800-1100 \mathrm{~mm}^{3}(+\mathrm{MG} ; n=6$, -MG; $n=4$ ). Collection of tumors for IHC staining was initiated two weeks post injection of FaDu cells and the collection periods lasted for two weeks. Two hours prior to euthanasia $0.06 \mathrm{mg} / \mathrm{g}$ pimonidazole was injected i.p.. Tumors were fixed in $4 \%$ formaldehyde for $24 \mathrm{~h}$, hereafter transferred to $70 \%$ ethanol, and finally embedded in paraffin and cut into $4 \mu \mathrm{m}$ slices. Each tumor was stained with the following antibodies; pimonidazole (PIMO; hypoxia) (HypoxyProbe-Omni Kit, HypoxyProbe Inc., Burlington, USA), Ki-67 (proliferation) (Dako; M7240), and CD31 (endothelial cell marker) (Abcam; ab28364). In addition haematoxylin eosin (HE) staining was performed.

Antibody concentrations were optimized on tumor samples from mice included in this study for optimal binding specificity. The following concentrations were used for analysis; PIMO 1:400, Ki-67 1:400, and CD31 1:50.

Deparaffination was performed by heating slides for $1 \mathrm{~h}$ at $40^{\circ} \mathrm{C}$, increasing temperature to $60^{\circ} \mathrm{C}$ and incubating for one additional hour. Slides were subsequently treated with xylene and rehydrated in descending concentrations of ethanol $(99,96,70 \%)$. Slides for Ki-67 antibody staining were furthermore exposed to microwave heating after rehydration to retrieve optimal binding. Endogenous peroxidase was blocked by Peroxidase Blocking Reagent (Dako, Glostrup, Denmark) for 8 min followed by Bovine Serum Albumin (BSA) blocking with $2 \%$ BSA for 10 min to avoid unspecific binding of antibodies. Primary antibody incubated for $1 \mathrm{~h}$ followed by secondary biotinylated EnVision FLEX ${ }^{\text {si }}$ (Dako, Glostrup, Denmark) incubation for $40 \mathrm{~min}$. Finally antibody staining was evoked by 3,3'-Diaminobenzidine (DAB) (Dako, Glostrup, Denmark) incubation for $10 \mathrm{~min}$ and counterstained with haematoxylin. Between all steps slides were rinsed in phosphate buffered saline (PBS, $0.2 \mathrm{M}, \mathrm{pH}=7.4$ ). After dehydration in increasing alcohol concentrations cover slides were mounted and slides scanned on an Axio scanner (Axio scan, Carl Zeiss, Germany). The Following parameters were analyzed; cell density, hypoxia percentages, micro vessel density (MVD), average vessel area, non-viable cell percentages, and proliferation percentages. Cell density and hypoxia was determined using the publicly available software Fiji (ImageJ). For nuclear density a nuclei count threshold of 50 pixels $^{2}$ to infinity was used (pixel size $0.022 \times 0.022 \mu \mathrm{m}$ ). The percentage of tumor hypoxia was evaluated using Color Deconvolution based on pimonidazole DAB-H staining. Based on constructed binary images (threshold between 210 and 220 RGB values of intensity) the percentage of hypoxia positive stained area in tumor slides was determined. MVD and average vessel area was determined using online image segmentation and endothelial cell analysis software CAIMAN (CAncer IMage ANalysis: http://www.caiman.org.uk) [18] in 5 selected ROIs. ROIs, excluding necrotic regions and artifacts, were manually drawn to represent entire slide (pixel size $0.088 \times 0.088 \mu \mathrm{m})$. Non-viable cell counts were determined in Fiji using the Advanced Weka Segmentation plug-in. Regions of viable cells, non-viable cells, and background in slide were marked to train the classifier and determine final segmentation. Calculation was made from the result image constructed by classifier. Proliferation in tumors was calculated using the online automated image analysis application ImmunoRatio (http:// jvsmicroscope.uta.fi/sites/default/files/software/immunoratio-plugin/index.html) [19]. Five defined ROIs representing entire slide, excluding necrosis and artifacts, were manually drawn and uploaded to define percentage of

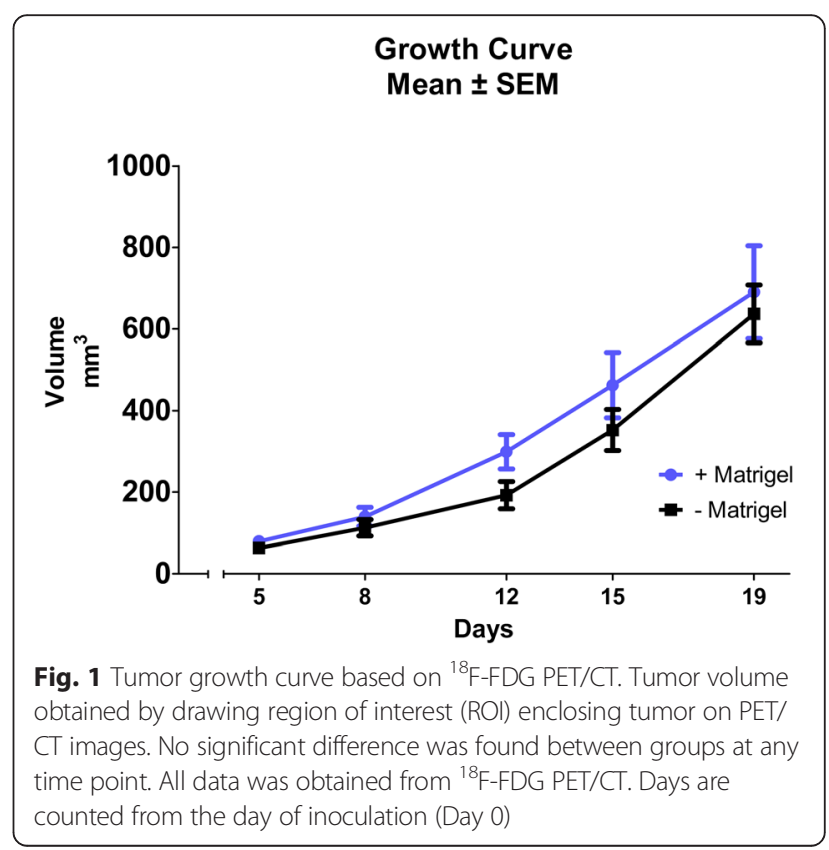




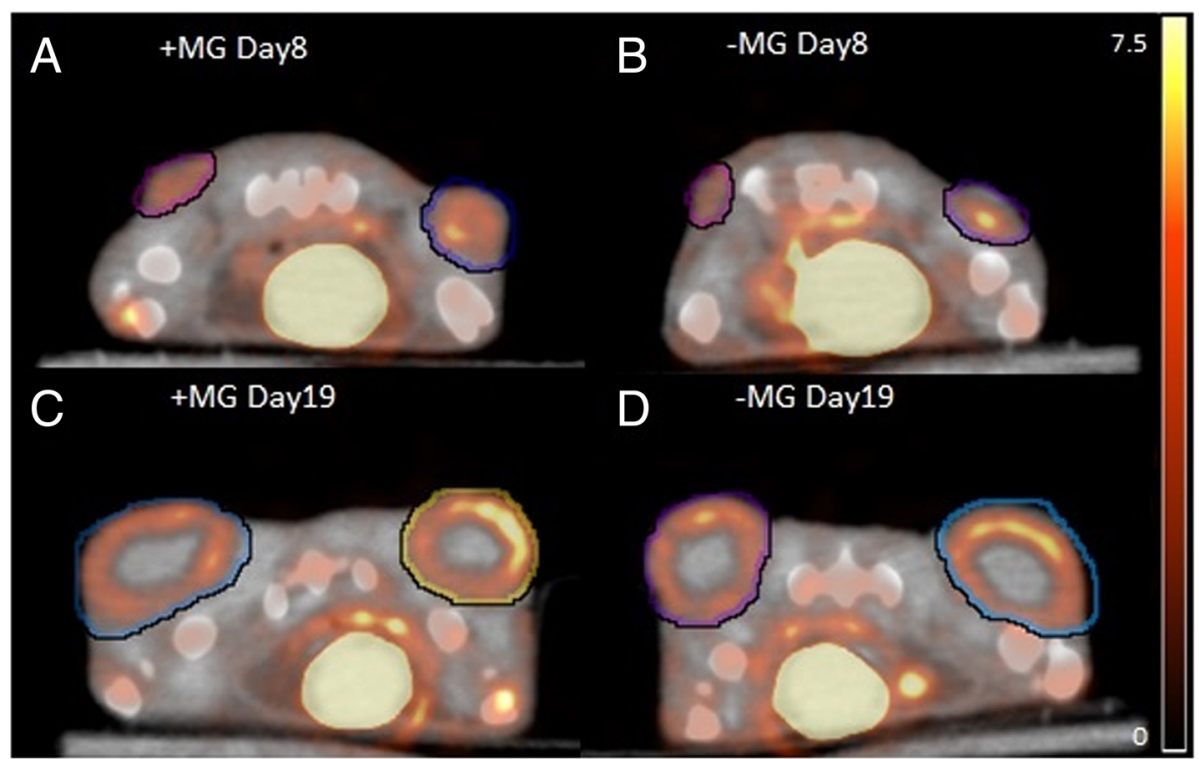

Fig. 2 Transverse section of an ${ }^{18} \mathrm{~F}$-FDG PET/CT image of mice with subcutaneous FaDu tumors. ${ }^{18} \mathrm{~F}$-FDG PET/CT scans $1 \mathrm{~h}$ after ${ }^{18} \mathrm{~F}$-FDG injection. Region of interests encapsulate tumor on each side of the flank. $\mathbf{a}$ and $\mathbf{c}+\mathrm{MG}$ mouse at scan day 8 and 19 , respectively. $\mathbf{b}$ and $\mathbf{d}-\mathrm{MG}$ mouse scan day 8 and 19, respectively. Scale bar is indicated in \%lD/g for all images calculated from a specific mass of $1 \mathrm{~g} / \mathrm{cm}^{3}$. Scan day represents number of days after inoculation
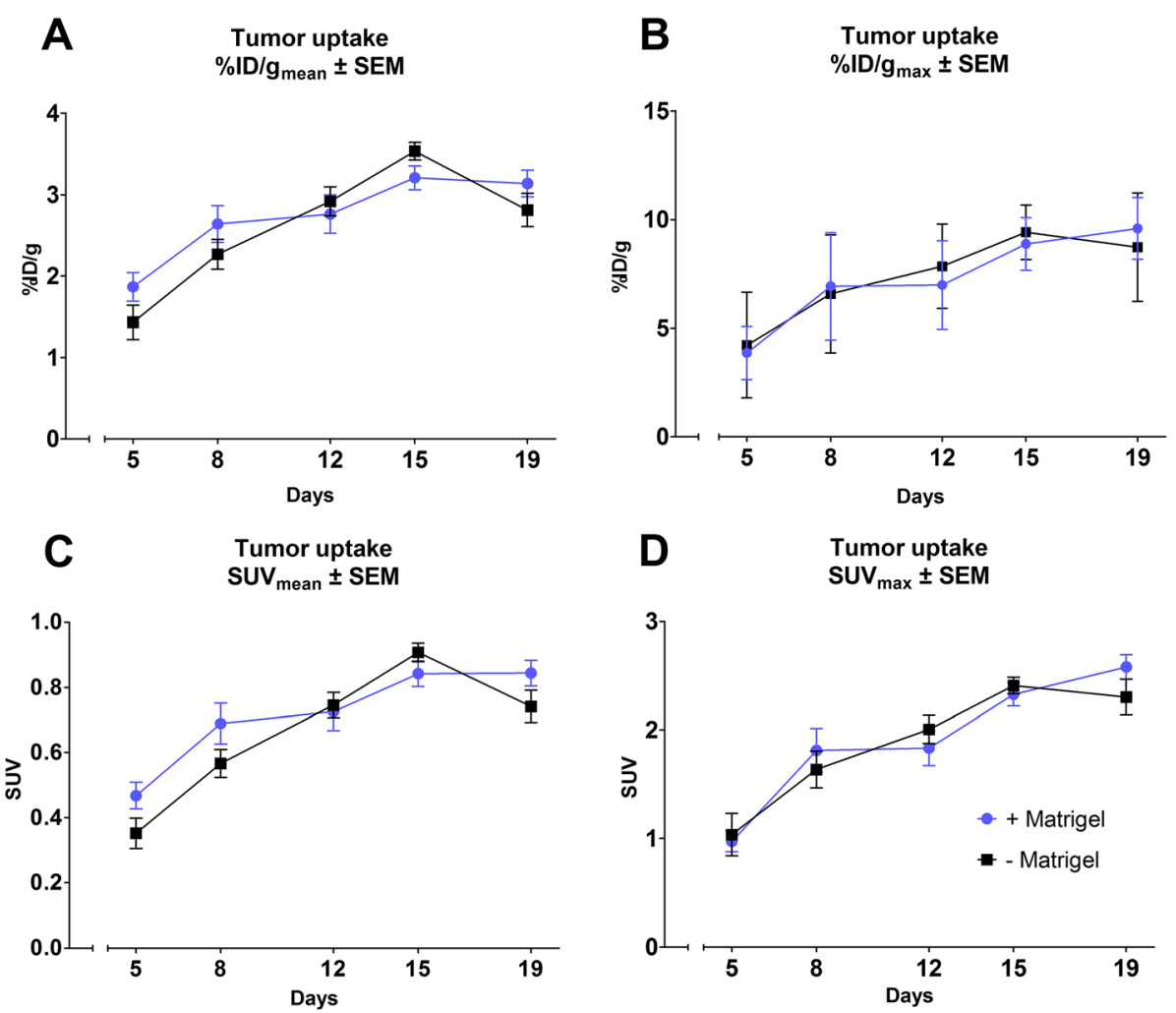

Fig. 3 Graphic presentation of tumor uptake results obtained from ${ }^{18}$ F-FDG PET/CT scan data. Uptake obtained from ROls drawn on tumor areas calculated as mean (a) and maximum (b) \%ID/g from a specific mass of $1 \mathrm{~g} / \mathrm{cm}^{3}$ and also as mean (c) and maximum (d) standardized uptake value (SUV). All data in plots obtained from ${ }^{18} \mathrm{~F}$-FDG PET/CT scans on Siemens Inveon Small Animal scanner $1 \mathrm{~h}$ post injection of tracer 
proliferating cells in total nuclei area (pixel size $0.088 \times$ $0.088 \mu \mathrm{m})$.

\section{Statistical analysis}

Statistical analysis was performed in GraphPad 6 (GraphPad Software, CA, USA). Comparison between groups of data from PET/CT scan was performed using Student's Ttest. Results are presented as Mean \pm SEM (Standard Error of Mean). Analysis of data from histological staining's was performed using One-Way ANOVA variance analysis with Holm-Sidak's post hoc test for multiple comparisons test to evaluate differences between groups of different tumor sizes. A p-value $<0.05$ was considered statistically significant in all tests.

\section{Results and discussion}

\section{Tracer uptake and tumor volume determined by ${ }^{18}$ F-FDG} $\mathrm{PET} / \mathrm{CT}$ scan

Data obtained from ${ }^{18} \mathrm{~F}$-FDG PET/CT identified no significant difference in FDG tumor uptake or tumor size between the $+\mathrm{MG}$ and -MG groups on each scan day, shown in Figs. 1 and 3. A tendency for increased growth rate of the tumors in the +MG group was observed between day 8 and 12 (Fig. 1), but growth was very compatible at later time points. Figure 2 illustrate ${ }^{18} \mathrm{~F}$-FDG $\mathrm{PET} / \mathrm{CT}$ images of mice from the different groups, and the compatible ${ }^{18} \mathrm{~F}$-FDG uptake can be readily appreciated at both time points. The observed compatibility of FDG uptake in tumors +/- MG indicates that ${ }^{18}$ F-FDG PET/ $\mathrm{CT}$ results are not influenced by the addition of $\mathrm{MG}$ for this tumor model (Fig. 3). SUV $\mathrm{Smax}_{\max }$ is often used clinically for classification of tumors in relation to staging, treatment, and response, and was therefore included in study to identify and evaluate maximum uptake in tumors [20]. No difference between $S_{U} V_{\max }$ in tumors with or without MG was found, indicating that MG, in this setup, does not influence maximum FDG uptake (Fig. 3d).

Previous studies have shown that tumor take in models are increased using MG [21]. In this study the take rate of tumors inoculated without MG was approximately $95 \%$ (32 out of 34 inoculated) and $100 \%$ (34 out of 34 inoculated) with MG, which indicates that MG does not influence tumor take for the investigated model.

The doubling in injection volume between groups might lead to a falsely determined tumor volume for the $+\mathrm{MG}$ group at early and intermediate time points, where a distinct part is mostly due to MG volume and not division of tumor cells. However, as seen in Fig. 1, tumor volume at
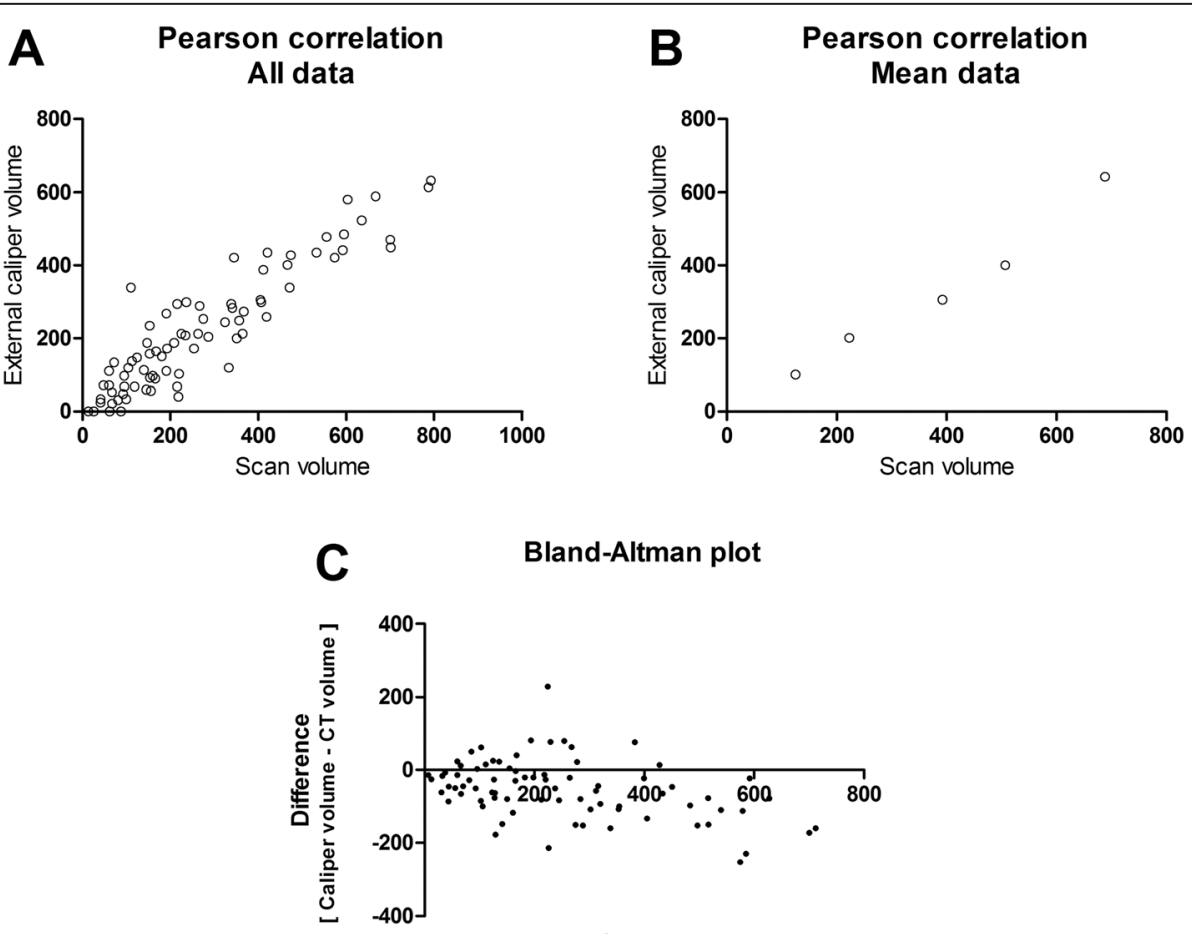

[ (Caliper volume + CT volume)/2 ]

Fig. 4 Pearson correlation model for ${ }^{18}$ F-FDG PET/CT determined tumor volume and external caliper determined tumor volume. Correlation between external caliper determined volumes and volumes determined from PET/CT scans. Pearson correlation model was used for the plotted values. a All scans. Correlation coefficient was 0.85 ( $p$-value $<0.0001)$. b Same regression model with mean value at each time point $(5,8,12,15$, and 19). Correlation coefficient was 0.98 ( $p$-value $=0.0017)$. $\mathbf{c}$ Bland-Altman plot showing the difference between measurements against the average to visualize bias between methods 
day 5 and 8 are virtually similar and the growth rates between these days follow the same development curve. Difference in growth rate was detected between day 8 and 12 (increase in rate for $+M G$ ). Grounds for injecting larger suspension volumes for the MG suspension was based on keeping the injected number of cells and concentration in media fixed, and from the results the difference in injection volume did not seem to have a distinct effect. Based on Figs. 1 and 3, it seems that the variation in size and tracer uptake is smaller in the -MG group compared to the +MG group. PET/CT scan data indicate that no significant advantage or drawback can be stated regarding MG use for tumor growth or tumor uptake in this model, but a decrease in group variation is observed for the -MG group. Based on this, there is little reason for using MG in FaDu xenograft models.

\section{Tumor development from IHC data}

IHC staining was made on tumors collected at different size groups in order to compare tumor microenvironment characteristics on different development stages. Size of collected tumors was defined using external caliper as described in the method section. To connect the two parts of the study in regard to tumor growth, Pearson correlation was performed between volumes determined from ${ }^{18} \mathrm{~F}$-FDG PET/CT scan and volumes determined from the external caliper (Fig. 4). A correlation coefficient of 0.85 (p-value $<0.0001$ ) was found for the correlation including all data points (Fig. 4a). Pearson correlation analysis based on mean values at each time point identified a correlation coefficient of 0.98 (p-value $=0.0017$ ) (Fig. 4b). Additionally, the validity of comaring tumor sizes between groups was found acceptable using Bland-Altman analysis (Fig. 4c).

Results obtained from IHC are presented in Fig. 5. One-way ANOVA test was used for the comparisons of characteristics between different size groups. IHC data showed that vascularization differed between the groups of the same tumor size. Statistical differences between micro vessel densities were found for tumor sizes; $500-700 \mathrm{~mm}^{3}$ ( $\mathrm{p}$-value $\left.=0.0457\right)$ and $800-1100 \mathrm{~mm}^{3}$ ( $\mathrm{p}$-value $=0.0214)$, and for average vessel area at 500$700 \mathrm{~mm}^{3}$ (p-value $=0.0237$ ) with the highest numbers for the -MG group (Fig. 5a and b). Angiogenesis was found to
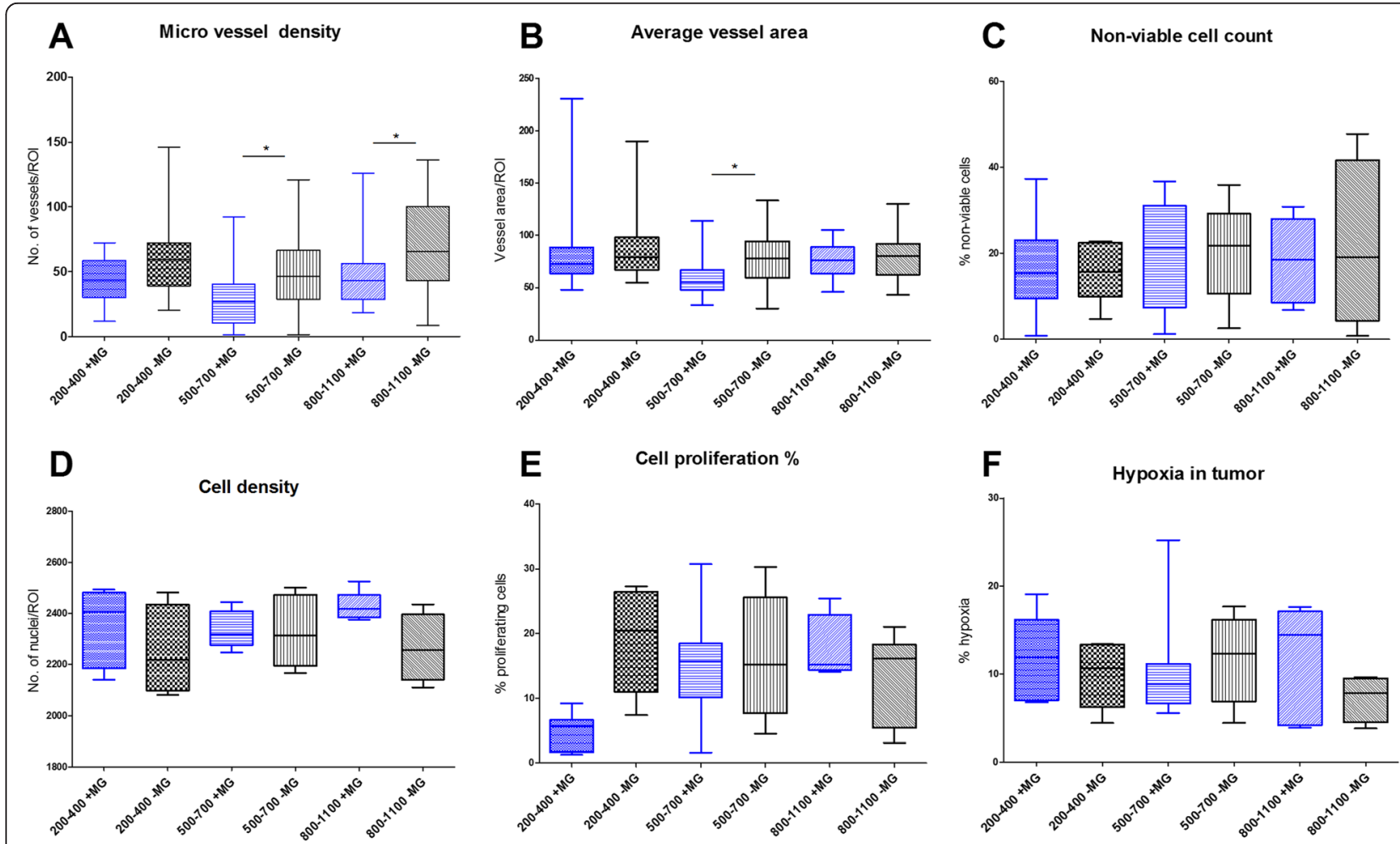

Fig. 5 Boxplot presentation of immunohistochemical results of collected tumors at different size stages. Results presented from all IHC data obtained. One way ANOVA with Holm-Sidak's post hoc test. a MVD as determined by CD31 staining. Significance found between groups of sizes $500-700 \mathrm{~mm}^{3}$ ( $p$-value $=0.0457$ ) and $800-1100 \mathrm{~mm}^{3}$ ( $p$-value $=0.0214$ ). MVD calculated as no. of vessels per ROI area. $\mathbf{b}$ Average vessel area incl. lumen from CD31 staining. Significant difference between groups in tumors size of 500-700 mm ( $p$-value $=0.024$ ). c Non-viable cell count presented from HE staining of slides. No significance found. $\mathbf{d}$ Cell density calculated from Haematoxylin staining illustrated, no significant difference found. e Cell proliferation determined as percent Ki-67 positive cells of total count. No statistical difference observed. $\mathbf{f}$ Percentages of hypoxia in tumor from PIMO antibody staining. No statistical difference found 
be significantly higher in tumors without MG. When comparing to the results of cell density, a tendency to a lower median in cell density for tumors without MG was observed. As discussed previously, tumor growth data obtained from PET/CT scans showed a tendency of increased tumor size for the +MG group at day 12-15 where tumors size was $200-400 \mathrm{~mm}^{3}$. Tumor cell proliferation showed a distinct difference for the small tumor size group (200-400 $\mathrm{mm}^{3}$ ) with a higher percentage of proliferating cells in the -MG group (Fig. 5e). These two observations are conflicting since the increased growth rate of the tumors in the +MG groups does not correlate with the proliferation percentage in collected tumors at same size. The tumor volume in the +MG group could, according to the proliferation percentages, hereby partly consist of MG volume but IHC results on other parameters contradicts this theory. Cell density in tumors of all sizes seemed to be rather similar, but as described previously, a lower median is observed for tumors in the -MG group. This hereby conversely describes the tumor area in $+M G$ to be denser in cell concentration than the -MG group. For the larger sized tumor groups proliferating cells in slides were compatible. In Fig. 6 examples of pre- and postanalysis images of IHC slides stained with CD31 (Fig. 6a+b), Ki-67 (Fig. 6c +d), and HE (Fig. 6e +f) are shown. From the IHC results MG seems to be without major influence on tumor development and the preclinical outcome in our model.

\section{Conclusions}

Our data indicate that using MG in cell suspension for inoculation induces no major impact on imaging and microenvironment characteristics of the $\mathrm{FaDu}$ hypopharyngeal carcinoma xenografts. Less variation is seen when no MG is used, which is in favor of not including MG in $\mathrm{FaDu}$ xenograft inoculation. Following the extensive use of the FaDu head and neck cancer xenograft model for PET imaging and tumor microenvironment characteristics, this study indicates that studies with and without MG use are comparable. Importantly, the observation in this study cannot be generally applied. Important differences potentially exist for human xenograft models other than FaDu in NMRI nude mice, which must be considered before comparing results between studies.

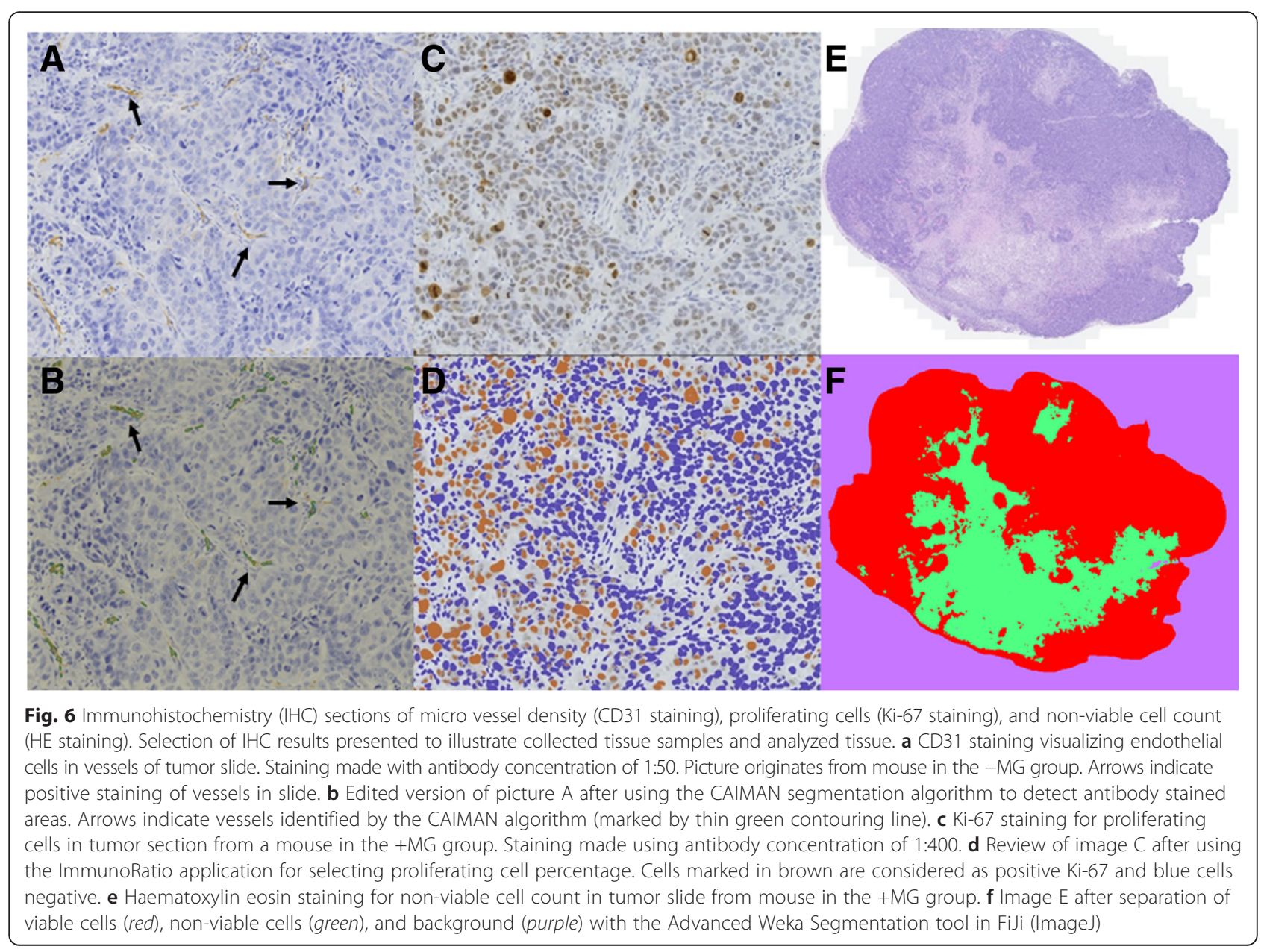




\section{Competing Interests}

The authors declare that they have no competing interests.

\section{Authors' contributions}

Frederikke P. Fliedner (FPF), Anders E. Hansen (AEH) and Andreas Kjaer (AK) designed the study. FPF, AEH and Jesper T. Jørgensen (JTJ) performed the experiments. All authors contributed to data analysis and interpretations. FPF drafted the manuscript and all authors provided input to revision of the manuscript and approved the final version.

\section{Acknowledgements}

Generous support from the Danish Cancer Society, the Lundbeck foundation, the Novo Nordisk Foundation, the Svend Andersen Foundation, the Innovation Fund Denmark, the Danish Research Council for Independent Research, Rigshospitalets Research Foundation, the Research Foundation of the Capital Region, the AP Møller Foundation, and the John and Birthe Meyer Foundation.

\section{Author details}

'Department of Clinical Physiology, Nuclear Medicine \& PET and Cluster for Molecular Imaging, Rigshospitalet and University of Copenhagen, Blegdamsvej 9, 2100 Copenhagen, Denmark. ${ }^{2}$ Department of Micro- and Nanotechnology, Center for Nanomedicine and Theranostics, DTU Nanotech, Technical University of Denmark, Building 423, 2800 Lyngby, Denmark.

\section{Received: 8 September 2015 Accepted: 4 January 2016}

\section{Published online: 14 January 2016}

\section{References}

1. Vukicevic S, Kleinman HK, Luyten FP, Roberts AB, Roche NS, Reddi AH. Identification of multiple active growth factors in basement membrane Matrigel suggests caution in interpretation of cellular activity related to extracellular matrix components. Exp Cell Res. 1992;202(1):1-8.

2. Kleinman HK, McGarvey ML, Hassell JR, Star VL, Cannon FB, Laurie GW, et al. Basement membrane complexes with biological activity. Biochemistry. 1986;25(2):312-8.

3. Fridman R, Benton G, Aranoutova I, Kleinman HK, Bonfil RD. Increased initiation and growth of tumor cell lines, cancer stem cells and biopsy material in mice using basement membrane matrix protein (Cultrex or Matrigel) co-injection. Nat Protoc. 2012;7(6):1138-44.

4. Benton G, Arnaoutova I, George J, Kleinman HK, Koblinski J. Matrigel: from discovery and ECM mimicry to assays and models for cancer research. Adv Drug Deliv Rev. 2014;79-80:3-18. doi:10.1016/j.addr.2014.06.005.

5. Arnaoutova I, George J, Kleinman HK, Benton G. Basement membrane matrix (BME) has multiple uses with stem cells. Stem Cell Rev. 2012;8(1):163-9.

6. Fridman R, Kibbey MC, Royce LS, Zain M, Sweeney M, Jicha DL, et al. Enhanced tumor growth of both primary and established human and murine tumor cells in athymic mice after coinjection with Matrigel. J Natl Cancer Inst. 1991;83(11):769-74.

7. Pretlow TG, Delmoro CM, Dilley GG, Spadafora CG, Pretlow TP. Transplantation of human prostatic carcinoma into nude mice in Matrigel. Cancer Res. 1991;51(14):3814-7.

8. Noel A, De Pauw-Gillet MC, Purnell G, Nusgens B, Lapiere CM, Foidart JM. Enhancement of tumorigenicity of human breast adenocarcinoma cells in nude mice by matrigel and fibroblasts. Br J Cancer. 1993;68(5):909-15.

9. Topley P, Jenkins DC, Jessup EA, Stables JN. Effect of reconstituted basement membrane components on the growth of a panel of human tumour cell lines in nude mice. Br J Cancer. 1993;67(5):953-8.

10. Hughes CS, Postovit LM, Lajoie GA. Matrigel: a complex protein mixture required for optimal growth of cell culture. Proteomics. 2010;10(9):1886-90.

11. Koshy M, Paulino AC, Howell R, Schuster D, Halkar R, Davis LW. F-18 FDG PET-CT fusion in radiotherapy treatment planning for head and neck cancer. Head Neck. 2005;27(6):494-502.

12. Baumann M, Liertz C, Baisch H, Wiegel T, Lorenzen J, Arps H. Impact of overall treatment time of fractionated irradiation on local control of human FaDu squamous cell carcinoma in nude mice. Radiother Oncol. 1994;32(2):137-43.

13. Menegakis A, Eicheler W, Yaromina A, Thames HD, Krause M, Baumann M. Residual DNA double strand breaks in perfused but not in unperfused areas determine different radiosensitivity of tumours. Radiother Oncol. 2011;100(1):137-44.
14. Schutze C, Bergmann R, Yaromina A, Hessel F, Kotzerke J, Steinbach J, et al. Effect of increase of radiation dose on local control relates to pre-treatment FDG uptake in FaDu tumours in nude mice. Radiother Oncol. 2007;83(3):311-5.

15. Tomayko MM, Reynolds CP. Determination of subcutaneous tumor size in athymic (nude) mice. Cancer Chemother Pharmacol. 1989;24(3):148-54.

16. Russell PJ, Raghavan D, Gregory P, Philips J, Wills EJ, Jelbart M, et al. Bladder cancer xenografts: a model of tumor cell heterogeneity. Cancer Res. 1986;46(4 Pt 2):2035-40.

17. Fueger BJ, Czernin J, Hildebrandt I, Tran C, Halpern BS, Stout D, et al. Impact of animal handling on the results of 18 F-FDG PET studies in mice. J Nucl Med. 2006:47(6):999-1006.

18. Reyes-Aldasoro CC, Griffiths MK, Savas D, Tozer GM. CAIMAN: an online algorithm repository for cancer image analysis. Comput Methods Programs Biomed. 2011;103(2):97-103.

19. Tuominen VJ, Ruotoistenmaki S, Viitanen A, Jumppanen M, Isola J. ImmunoRatio: a publicly available web application for quantitative image analysis of estrogen receptor (ER), progesterone receptor (PR), and Ki-67. Breast Cancer Res. 2010;12(4):R56.

20. Paidpally V, Chirindel A, Lam S, Agrawal N, Quon H, Subramaniam RM. FDG-PET/CT imaging biomarkers in head and neck squamous cell carcinoma. Imaging Med. 2012;4(6):633-47.

21. Mullen P, Ritchie A, Langdon SP, Miller WR. Effect of Matrigel on the tumorigenicity of human breast and ovarian carcinoma cell lines. Int J Cancer. 1996;67(6):816-20.

\section{Submit your next manuscript to BioMed Central and we will help you at every step:}

- We accept pre-submission inquiries

- Our selector tool helps you to find the most relevant journal

- We provide round the clock customer support

- Convenient online submission

- Thorough peer review

- Inclusion in PubMed and all major indexing services

- Maximum visibility for your research

Submit your manuscript at www.biomedcentral.com/submit
Biomed Central 